Research paper

\title{
Safety and immunogenicity of a Recombinant Stabilized Prefusion SARS- CoV-2 Spike Protein Vaccine (MVC-COV1901) Adjuvanted with CpG 1018 and Aluminum Hydroxide in healthy adults: A Phase 1, dose- escalation study
}

\author{
Szu-Min Hsieh ${ }^{\mathrm{a}}$, Wang-Da Liu ${ }^{\mathrm{a}}$, Yu-Shan Huang ${ }^{\mathrm{a}}$, Yi-Jiun Lin ${ }^{\mathrm{b}}$, Erh-Fang Hsieh ${ }^{\mathrm{b}}$, \\ Wei-Cheng Lian ${ }^{\mathrm{b}}$, Charles Chen ${ }^{\mathrm{b}, \mathrm{c}}$, Robert Janssen ${ }^{\mathrm{d}}$, Shin-Ru Shih ${ }^{\mathrm{e}}$, Chung-Guei Huang, ${ }^{\mathrm{e}, \mathrm{f}}$, \\ I-Chen Tai ${ }^{\mathrm{b}, *}$, Shan-Chwen Chang ${ }^{\mathrm{a}, *}$ \\ a Section of Infectious Diseases, Division of Infectious Diseases, Department of Internal Medicine, National Taiwan University Hospital and College of Medicine, \\ National Taiwan University, Taiwan \\ ${ }^{\mathrm{b}}$ Medigen Vaccine Biologics Corp., Taiwan

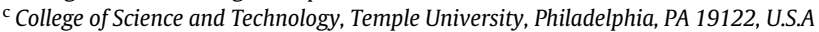 \\ d Dynavax Technologies Corporation, Emeryville, CA 94608, U.S.A \\ e Research Center for Emerging Viral Infections, College of Medicine, Chang Gung University, Taoyuan City, Taiwan \\ ${ }^{\mathrm{f}}$ Department of Laboratory Medicine, Linkou Chang Gung Memorial Hospital, Taoyuan City, Taiwan
}

\section{A R T I C L E I N F O}

\section{Article History:}

Received 21 April 2021

Revised 8 June 2021

Accepted 10 June 2021

Available online 26 June 2021

\begin{abstract}
A B S T R A C T
Background: This was a phase 1, dose-escalation open-label trial to evaluate the safety and immunogenicity of MVC-COV1901, a SARS-CoV-2 S-2P protein vaccine adjuvanted with aluminum hydroxide and CpG 1018.

Methods: Between September 28 and November 13 2020, 77 participants were screened. Of these, 45 healthy adults from 20 to 49 years of age were to be administered two doses of MVC-COV1901 in doses of $5 \mu \mathrm{g}, 15 \mu \mathrm{g}$, or $25 \mu \mathrm{g}$ of spike protein at 28 days apart. There were 15 participants in each dose group; all were followed for 28 days after the second dose at the time of the interim analysis. Adverse events and laboratory data were recorded for the safety evaluation. Blood samples were collected for humoral, and cellular immune response at various time points. Trial Registration: ClinicalTrials.gov NCT 04487210.

Findings: Solicited adverse events were mostly mild and similar. No subject experienced fever. After the second dose, the geometric mean titers (GMTs) for SARS-CoV-2 spike-specific immunoglobulin G were 7178.2, 7746.1, 11,220.6 in the $5 \mu \mathrm{g}, 15 \mu \mathrm{g}$, and $25 \mu \mathrm{g}$ dose groups, respectively. The neutralizing activity were detected in both methods. (Day 43 GMTs, 538.5, 993.1, and 1905.8 for pseudovirus; and 33.3, 76.3, and 167.4 for wild-type virus). The cellular immune response induced by MVC-COV1901 demonstrated substantially higher numbers of IFN- $\gamma$ - producing cells, suggesting a Th1-skewed immune response.

Interpretation: The MVC-COV1901 vaccine was well tolerated and elicited robust immune responses and is suitable for further development.

Funding: Medigen Vaccine Biologics Corporation

(c) 2021 The Author(s). Published by Elsevier Ltd. This is an open access article under the CC BY-NC-ND license
\end{abstract}

(http://creativecommons.org/licenses/by-nc-nd/4.0/)

\section{Introduction}

Human infections due to SARS-CoV-2 began to spread globally following the outbreak in Wuhan, China. WHO declared the COVID-19

\footnotetext{
* Corresponding author.

E-mail addresses: kathytai@medigenvac.com (I.-C. Tai), changsc@ntu.edu.tw (S.-C. Chang).
}

outbreak as a public health emergency and subsequently characterized it as a pandemic on March $11^{\text {th }}, 2020$ [1].

Approximately $15 \%$ of COVID-19 cases are severe requiring oxygen support and $5 \%$ are critical with complications such as respiratory failure, acute respiratory distress syndrome (ARDS), sepsis, and septic shock [2].

There is currently no cure for the potentially lethal COVID-19 making development of a range of vaccines imperative. For vaccines 


\section{Research in context}

\section{Evidence before this study}

We searched PubMed, up to Feb 28, 2021, for published clinical trials assessing the safety and immunogenicity of COVID-19 vaccines. The searched terms used were COVID-19, SARS-CoV$2, \mathrm{~S}-2 \mathrm{P}$, recombinant protein, "Clinical trials" and "vaccine". We identified no published clinical trials of the safety and immunogenicity of COVID-19 vaccine using S-2P recombinant protein.

\section{Added value of this study}

We are reporting interim results of a phase 1 , dose-escalation study designed to evaluate the safety and immunogenicity of the COVID-19 vaccine, MVC-COV1901. This vaccine consists of the S-2P protein, developed by Dr. Barney S. Graham at Vaccine Research Center, National Institute of Allergy and Infectious Diseases (NIAID), U.S.A., using a recombinant protein platform in combination with the adjuvants CpG 1018, a Toll-like receptor 9 agonist, and aluminum hydroxide. This study demonstrates that MVC-COV1901 was well tolerated with most solicited post-injection reactions reported as mild. The neutralizing antibody Geometric Mean Titers (GMT) were comparable with the GMT levels of human convalescent sera.

\section{Implications of all the available evidence}

These immunogenicity results were comparable with human convalescent serum panel in this study, and with favorable tolerability. This vaccine candidate can be stored at $2-8{ }^{\circ} \mathrm{C}$. We believe that subunit vaccines play an indispensable role against the SARS-CoV-2 pandemic due to their tolerability and possible broader indications for vulnerable populations. The article is the first clinical report of a subunit protein vaccine using S-2P antigen, adjuvanted with Th1-skewing $\mathrm{CpG}$ and aluminum hydroxide that demonstrates good tolerability and immunogenicity, and support further clinical development of S-2P protein adjuvanted with CpG 1018 and aluminum hydroxide.

intended to generate a protective immune response, using an antigen with proper conformation is critical. The neutralizing antibodies induced by the spike $(S)$ protein block viruses from binding to their target receptor ACE2 and hence inhibit viral infection. The S protein has two major conformational states, prefusion and postfusion [3]. The $\mathrm{S}-2 \mathrm{P}$ protein is a recombinant version of the $\mathrm{S}$ protein developed by McLellan and Graham and is a stabilized prefusion $S$ ectodomain, encoding residues $1-1208$ of the SARS-CoV-2 spike protein with two proline substitutions at residues 986 and 987, a "GSAS" substitution at residues $682-685$ to abolish the furin cleavage site, and a T4 fibritin trimerization motif at the C-terminus [4]. Cryo-electron microscopy showed the protein produced by this construct is in the prefusion conformation and can bind to ACE2 [4]. A similar strategy had been used to maintain the MERS-COV S protein in the prefusion conformation and demonstrated that the stabilized MERS-COV S protein was able to elicit high neutralizing antibody concentrations [5]. Additionally, mRNA vaccines coding for the S-2P protein of SARSCoV-2 have proven to be efficacious [6]. Medigen's MVC-COV1901 vaccine is formulated as S-2P adjuvanted with Dynavax's CpG 1018 and aluminum hydroxide. CpG 1018 is an oligodeoxynucleotide which acts as a toll-like receptor 9 agonist and has been shown in our preclinical studies to enhance immunogenicity and induce a Th1-skewed immune response [7]. We conducted a first-in-human, phase 1, dose-escalation study done to assess the safety, and immunogenicity of three dose levels of MVC-COV1901 administered to healthy adults as two doses 28 days apart.

\section{Methods}

\subsection{Trial design}

This study was a phase 1, prospective, open-label, dose-escalation, non-randomized study to evaluate the safety and immunogenicity of a SARS-CoV-2 vaccine in adults aged 20 to 49 years. The study was initiated at the National Taiwan University Hospital, a medical center located in northern Taiwan, in September 2020. The trial protocol and informed consent form were approved by the Taiwan Food and Drug Administration and the ethics committee at the site. The trial was conducted in accordance with the principles of the Declaration of Helsinki and Good Clinical Practice. An independent data and safety monitoring board (DSMB) was established to monitor safety data and the trial conduct. (ClinicalTrials.gov NCT 04487210) The study fully adhered to the protocol and statistical analysis plan before this interim analysis. The protocol is available in the appendix.

\subsection{Participants}

The study aimed to enroll 45 subjects. The number of subject was based on the phase 1 design of Moderna[8] and was considered adequate to obtain a preliminary assessment of safety and immunogenicity of three different dose levels of antigen to identify a dose level to move forward into a phase 2 study. Eligible participants were healthy adults 20 to 49 years of age. Eligibility was determined based on medical history, physical examination, laboratory tests, and investigators' clinical judgment. Exclusion criteria included a history of known potential exposure to SARS CoV-1 or 2 viruses, having received any other COVID-19 vaccine, impaired immune function, history of autoimmune disease, uncontrolled HIV, HBV, or HCV infection, abnormal autoantibody tests, febrile or acute illness within 2 days of first dose, and acute respiratory illness within 14 days of first dose.

\subsection{The investigational vaccine}

We applied technology previously used for MERS-CoV to produce a prefusion-stabilized SARS-CoV-2 spike protein, S-2P [4], adjuvanted with CpG 1018 and aluminum hydroxide as previous reported [7]. Each MVC-COV1901 vaccine contained 5, 15, or $25 \mu \mathrm{g}$ of S-2P adjuvanted with $750 \mu \mathrm{g}$ of $\mathrm{CpG} 1018$ and $375 \mu \mathrm{g}$ (Al equivalent to weight) of aluminium hydroxide, administered as a single $0.5 \mathrm{~mL}$ intramuscular (IM) injection. The vaccine was produced in the Medigen Vaccine Biologics Corporation facility which is compliant with the current good manufacturing practices (cGMP).

\subsection{Interventions}

This study was a dose escalation study with three separate groups of participants 20 to 49 years of age. Each sub-phase consisted of 15 participants. The three different dose levels employed were 5, 15, and $25 \mu \mathrm{g}$ of S-2P protein for cohort $1 \mathrm{a}, 1 \mathrm{~b}$, and 1c, respectively. The vaccination schedule consisted of two doses, administered by IM injection in the deltoid muscle of the non-dominant arm 28 days apart, on Day 1 and Day 29. The protocol permitted an interim analysis to facilitate decisions regarding vaccine development strategy.

Cohort 1a: Four sentinel participants were to be recruited to receive vaccine with $5 \mu \mathrm{g}$ of S-2P to evaluate the preliminary safety data of the vaccine. If no $\geq$ Grade 3 Adverse Event (AE) or Serious Adverse Event (SAE) occurred within 7 days after the first dose in the 4 sentinel participants, dosing of the remaining participants in Phase $1 \mathrm{a}$ and Phase $1 \mathrm{~b}$ would proceed. 
Cohort 1b: Another 4 sentinel participants were to be enrolled to receive vaccine with $15 \mu \mathrm{g}$ of S-2P If no $\geq$ Grade $3 \mathrm{AE}$ or SAE occurred within 7 days after the first dose in the 4 sentinel participants, dosing of the remaining participants in Phase $1 \mathrm{~b}$ and Phase 1c would proceed.

Cohort 1c: Another 4 sentinel participants would be enrolled to receive vaccine with $25 \mu \mathrm{g}$ of S-2P If no $\geq$ Grade $3 \mathrm{AE}$ or SAE occurred within 7 days after the first dose in the 4 sentinel participants, dosing of the remaining participants in Phase 1c would proceed.

If any $\geq$ Grade $3 \mathrm{AE}$ or SAE occurred, the DSMB was to evaluate preliminary safety data and determine if it was safe to continue dosing.

An interim analysis of immunogenicity and safety data was to be conducted when all participants completed the visit at 28 days after the second dose.

\subsection{Outcomes}

The primary endpoint was to evaluate the safety of MVC-COV1901 in three different strengths (5, 15, and $25 \mu \mathrm{g} \mathrm{S}-2 \mathrm{P}$ protein adjuvanted with $\mathrm{CpG} 1018$ and aluminium hydroxide) from Day 1 to 28 days after the second dose. Vital signs and electrocardiogram (ECG) were performed before and after vaccination. Participants were observed for at least $30 \mathrm{~min}$ after each dose to identify any immediate AEs, and were asked to record solicited local (pain, erythema, swelling/induration) and systemic (fever, myalgia, malaise/ fatigue, nausea/vomiting, diarrohea) AEs in the participant's diary card for up to 7 days after each dose. Unsolicited AEs were recorded for 28 days following each dose; all other AEs, SAEs and adverse events of special interest (AESIs) were recorded throughout the study period (approximately 7 months). Please refer to protocol for more details.

Serum samples were collected for haematology, biochemistry and immunology evaluation measured as described in the protocol.

The immunogenicity endpoints were to evaluate neutralizing antibody titers and binding antibody titers at 14 days (Day 15) and 28 days (Day 29) after first and at 14 days (Day 43) and 28 days (Day 57) after second dose, as well as 90 days and 180 days after the second dose. Convalescent serum specimens from 35 recovered COVID19 patients (Mitek COVID-19 Panel 1.1 and COVID-19 Panel 1.4 obtained from Access Biologicals LLC) were also tested. (See Table S1 for characteristics of the patients who provided convalescent sera.) Cellular immune responses were evaluated at 28 days after the second dose by interferon-gamma (IFN- $\gamma$ ) ELISpot and interleukin (IL)4 ELISpot.

\subsection{Laboratory methods}

SARS-CoV-2 spike-specific immunoglobulin G (IgG): Total serum anti-Spike IgG titers were detected with direct enzyme-linked immunosorbent assay (ELISA) using customized 96-well plates coated with S-2P antigen.

SARS-CoV-2 pseudovirus neutralization assay: Pseudovirus production and titration followed the previous report [7]. Serial dilutions of the samples to be tested were performed (initial dilution of 1:20 followed by two-fold dilutions to a final dilution of 1:2560). The diluted serum was mixed with an equal volume of pseudovirus (1000 TU) and incubated before adding to the plates with HEK293-hAce2 cells $\left(1 \times 10^{4}\right.$ cells/well). The amount of pseudovirus entering the cells was calculated by lysing and measuring the relative luciferase units (RLU). Fifty percent inhibition dilution (concentration) titers $\left(\mathrm{ID}_{50}\right)$ were calculated considering uninfected cells as $100 \%$ neutralization and cells transduced with virus as $0 \%$ neutralization and reciprocal $\mathrm{ID}_{50}$ geometric mean titers (GMT) were both determined.

Wild-type SARS-CoV-2 neutralization assay: SARS-CoV-2 virus (hCoV-19/Taiwan/CGMH-CGU-01/2020, GenBank accession
MT192759) was titrated to obtain TCID $_{50}$ and Vero E6 cells $\left(2.5 \times 10^{4}\right.$ cells/well) were seeded in 96-well plates and incubated. The sera underwent two-fold dilutions with the final dilution being 1:8192, and the diluted sera were mixed with equal volume of viral solution containing $100 \mathrm{TCID}_{50}$. The serum-virus mixture was incubated and then added to the plates containing the Vero E6 cells, followed by further incubation. The neutralizing titer was defined as the reciprocal of the highest dilution capable of inhibiting 50\% of cytopathic effect

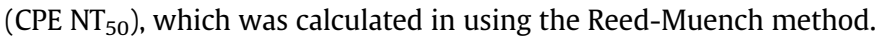
The National Institute for Biological Standards and Control (NIBSC; Potters Bar, UK) reference serum sample 20/130, was analysed using the same validated assays as a comparator.

Cellular immune response: The number of antigen-specific IFN- $\gamma$ or IL-4 secreting Spot Forming Units (SFU) were determined by ELISpot assays. Cryopreserved peripheral blood mononuclear cells (PBMC) were rapidly thawed and allowed to rest overnight. Cells were dispensed at $1 \times 10^{5}$ cells per well for IFN- $\gamma$ ELISpot assay (Human IFN- $\gamma$ ELISpot Kit, MABTECH) or $2 \times 10^{5}$ cells per well for IL4 ELISpot assay (Human IFN- $\gamma$ ELISpot Kit, MABTECH). Cells were stimulated with a pool of peptides consisting mainly of 15-mer sequences with 11 amino acids overlap, covering the N-terminal S1 domain of the $\mathrm{S}$ protein of SARS-CoV-2 (PepTivator SARS-CoV-2 Prot_S1, Miltenyi Biotec) and incubated at $37{ }^{\circ} \mathrm{C}$ for $24-48$ h. Cells stimulated with CD3-2 mAb served as the positive control. IFN- $\gamma$ or IL-4 release were detected following the manuals and the spots were counted using the CTL automatic ELISpot reader. The mean SFU counted in peptide pool stimulation triplicate was calculated and normalized by subtracting the mean of the negative control replicates (control media). Results were expressed as SFU per million PBMC.

\section{Statistical analysis}

The sample size was not based on any statistical hypothesis. Only descriptive statistics are presented for all variables defined by group. Safety analyses were performed on the Total Vaccinated Group (TVG) population who received at least 1 dose of vaccine. The immunogenicity endpoints comprised the Geometric Mean Titer (GMT) and Sero-conversion Rate (SCR) of antigen specific immunoglobulins and wild type virus and pseudovirus neutralizing antibody titers. SCR is defined as the percentage of participants with $\geq 4$-fold increase in titers from the baseline or from half of the lower limit of detection (LoD) if undetectable at baseline. The GMT and SCR are presented with two-sided 95\% CI. Antigen specific cellular immune responses are presented as means determined by IFN- $\gamma$ ELISpot and IL-4 ELISpot.

\subsection{Role of the funding source}

Taiwan Centers for Disease Control, Ministry of Health and Welfare provided grant funding for this study, but does not necessarily stand by any commentary made in this paper.

Medigen Vaccine Biologics Corp. was the study sponsor and manufacturer of the investigational vaccine, and co-designed the trial, provided the study product, and coordinated interactions with regulatory authorities. The sponsors used contract clinical research organization to oversee clinical site operations. Data were collected by the clinical site research staff, managed by a contract research organization data management team, monitored by a contract research organization, and overseen by the sponsor and an independent data and safety monitoring board. The interim analysis was performed by the contract research organization. Data interpretation, manuscript preparation were performed by the authors and the decision to submit the manuscript for publication was made by the authors. 


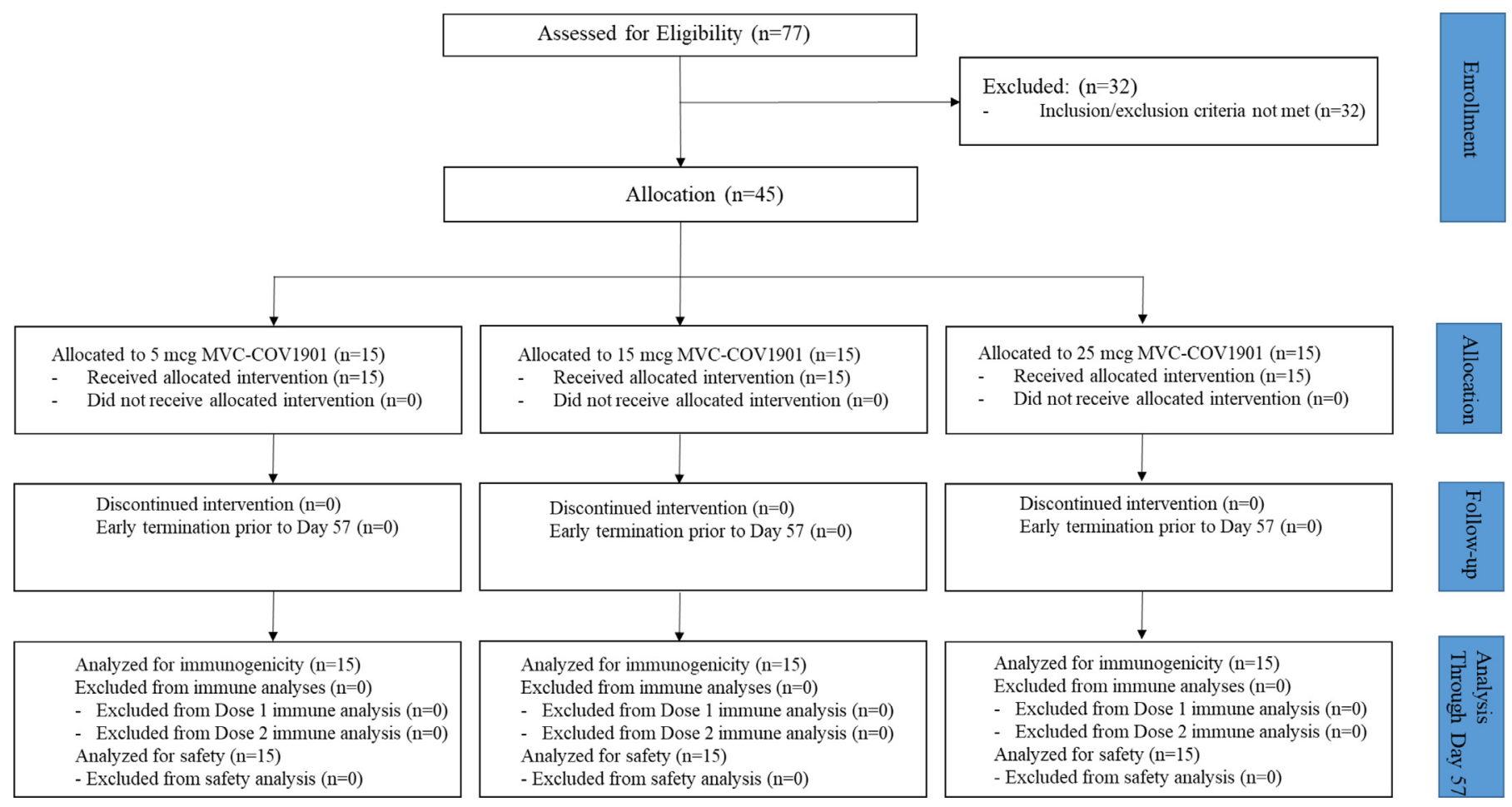

Fig. 1. Consort Flow Diagram.

Table 1

Demographic Characteristics of Eligible Participants.

\begin{tabular}{lllll}
\hline & LD: $5 \mu \mathrm{g}$ & MD: $15 \mu \mathrm{g}$ & HD: $25 \mu \mathrm{g}$ & Total \\
\hline $\begin{array}{l}\text { No. of Participants } \\
\text { Age }\end{array}$ & 15 & 15 & 15 & 45 \\
$\begin{array}{l}\text { Mean (SD), years } \\
\text { Gender }\end{array}$ & $36.7(8.97)$ & $33.3(8.03)$ & $31.5(5.78)$ & $33.8(7.84)$ \\
$\begin{array}{l}\text { Male, No. (\%) } \\
\text { Female, No. (\%) }\end{array}$ & $7(46.7 \%)$ & $9(60.0 \%)$ & $12(80.0 \%)$ & $28(62.2 \%)$ \\
$\begin{array}{l}\text { BMI (kg/m }) \\
\text { Mean (SD) }\end{array}$ & $23.18(3.394)$ & $6(40.0 \%)$ & $3(20.0 \%)$ & $17(37.8 \%)$ \\
\hline
\end{tabular}

\section{Results}

Between September 28 and November 13 2020, 77 participants were screened. Of these, 45 eligible participants completed two doses of MVC-COV1901 (Fig. 1). Most screen failures were due to abnormal autoantibody tests (defined antinuclear antibody titer $\geq 1: 40$ as abnormal). Participants' baseline demographic characteristics are summarized in Table 1.

\subsection{Safety}

No SAE or AESI occurred at this data cut-off point (December 23, 2020). No study intervention was modified or interrupted. Occurrences of solicited AEs are summarized in Fig. 2 and Table S2. The most commonly reported local AEs were pain/tenderness $(80.0 \%)$, while malaise/fatigue (28.9\%) were the most commonly reported systemic AEs among all treatment groups. All local and systemic AEs were mild, except for one malaise/fatigue in the $25 \mu \mathrm{g}$ dose group. No participant had fever. Solicited AEs after the first and the second dose were similar. Evaluation of safety laboratory values, ECG interpretation, and other unsolicited adverse events revealed no specific concern (Table S3). An increase in anti-nuclear antibody occurred in $31.1 \%$ of participants but all titers were all $<1$ : 160 and all were asymptomatic.

\subsection{Humoral immune response}

The humoral immunogenicity results are summarized in Fig. 3 and Table S4.

Binding IgG titers to $S$ protein increased rapidly after the second dose, with seroconversion in all participants by Day 43 and 57 . The GMTs peaked at Day 43 with a value of 7178.2 (95\% CI: 4240.3-12,151.7), 7746.1 (95\% CI: 5530.2-10,849.8), 11,220.6 (95\% CI: 8592.293-14,652.84) in the $5 \mu \mathrm{g}, 15 \mu \mathrm{g}$, and $25 \mu \mathrm{g}$ dose groups, respectively. The GMT levels in the $5 \mu \mathrm{g}, 15 \mu \mathrm{g}$, and $25 \mu \mathrm{g}$ dose groups on Day 43 ranged from 3.3 to 5.1 times the GMT of convalescent serum specimens. (2179.6, [95\%CI: 1240.9 - 3828.4]) (Fig. 3A and Table S4).

No subject had detectable pseudovirus neutralizing titers $\left(\mathrm{ID}_{50}\right)$ at the lower limit of serum concentration tested (1:20 dilution) in the assay at baseline. At Day 43, the pseudovirus neutralizing titers $\left(\mathrm{ID}_{50}\right)$ showed peak GMTs of 538.5 (95\% CI: 261.9 - 1107.0), 993.1 (95\% CI: 655.0-1505.7), and 1905.8 (95\% CI: $1601.7-2267.8$ ) in the $5 \mu \mathrm{g}$, $15 \mu \mathrm{g}$, and $25 \mu \mathrm{g}$ dose groups, respectively. All participants seroconverted after the second dose. The GMT levels in the $5 \mu \mathrm{g}, 15 \mu \mathrm{g}$, and $25 \mu \mathrm{g}$ dose groups on Day 43 ranged from 1.25 to 4.4 times the GMT of convalescent serum specimens. (430.5, [95\%CI: 274.9-674.0]) (Fig. 3B and Table S4).

Before vaccination, no subject had detectable wild-type virus neutralizing titers $\left(\mathrm{NT}_{50}\right)$ at the lower limit of serum concentration tested (1:8 dilution) in the assay. After the second dose, neutralizing responses were identified in serum samples from all participants in the $15 \mu \mathrm{g}$ and $25 \mu \mathrm{g}$ dose groups. At Day 43, the GMTs were 33.3 (95\% CI: 18.5-59.9), 76.3 (95\% CI: 53.7-108.3), and 167.4 (95\% CI: 122.1-229.6) in the $5 \mu \mathrm{g}, 15 \mu \mathrm{g}$, and $25 \mu \mathrm{g}$ dose groups, respectively. At Day 57, GMTs were similar in the $15 \mu \mathrm{g}$ and $25 \mu \mathrm{g}$ dose groups: 52.2 (95\% CI: 37.9 - 71.8) and 81.9 (95\% CI: 55.8 - 120.2), respectively. The GMT levels in the $5 \mu \mathrm{g}, 15 \mu \mathrm{g}$, and $25 \mu \mathrm{g}$ dose groups on Day 43 were $0.8,1.8$, and 3.9 times the GMT of convalescent serum specimens (42.7, [95\%CI: 26.4 - 69.0]; titers ranged from undetected to 631.0). All participants in $15 \mu \mathrm{g}$ and $25 \mu \mathrm{g}$ dose groups seroconverted at Day 43 and Day 57; some were similar to 


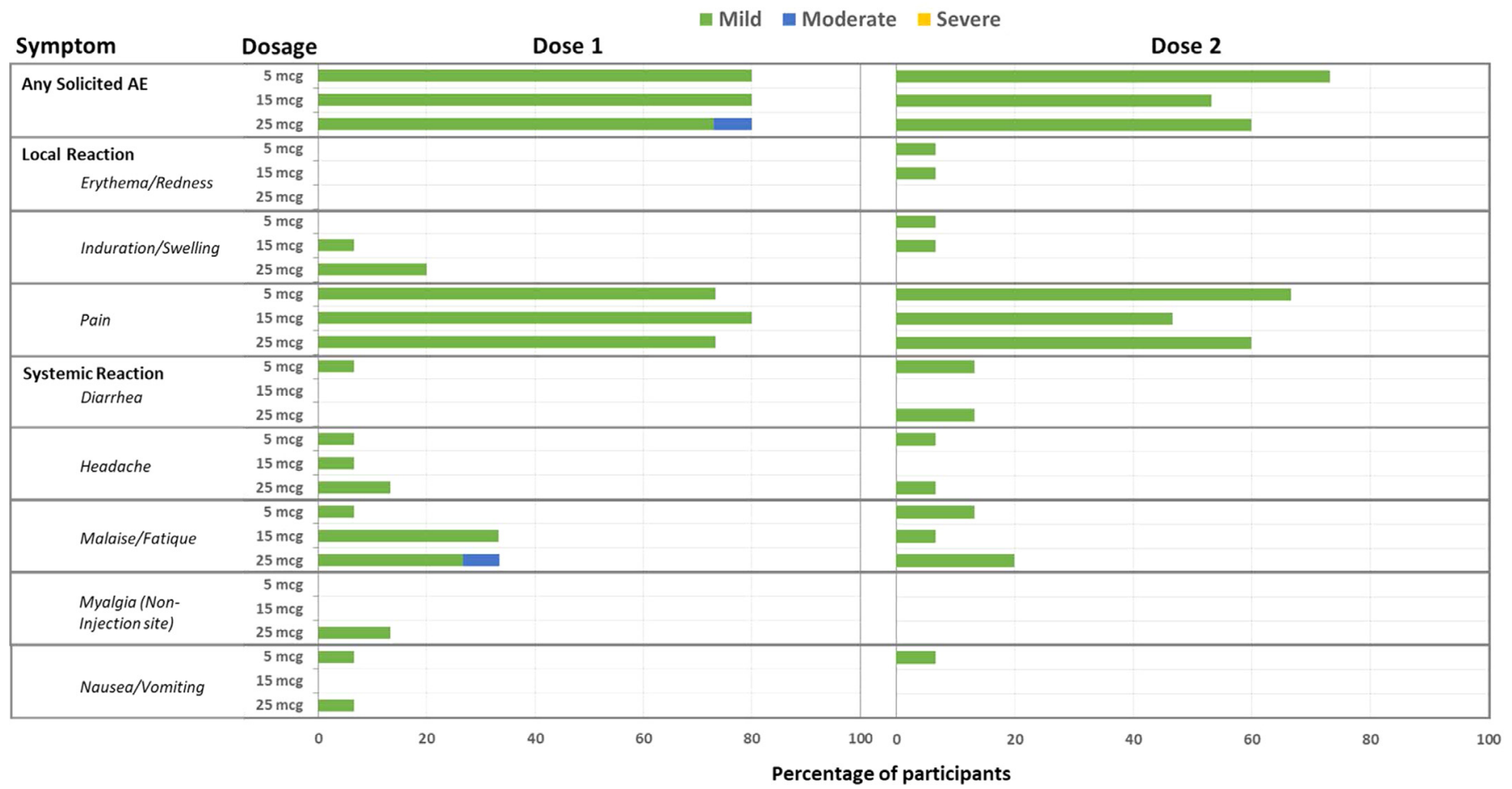

Fig. 2. Summary of Solicited Adverse Events

Participants were asked to record solicited local and systemic adverse events in the participant's diary card for up to 7 days after each vaccination. Solicited AEs were tabulated and graded as mild, moderate, or severe.

the NIBSC reference serum 20/130 (281.8). The results of wild-type SARS-CoV-2 neutralizing antibody titers are summarized in Fig. $3 \mathrm{C}$ and Table S4.

\subsection{Cellular immune response}

All participants had minimal IFN- $\gamma$ secreting T cells at baseline. By Day 57, a mean of $161.3,85.5$ and 94.9 IFN $-\gamma$ secreting T cells were observed per million cells in participants vaccinated with $5 \mu \mathrm{g}$, $15 \mu \mathrm{g}$, and $25 \mu \mathrm{g}$, respectively.

Before vaccination, all participants had minimal IL-4 secreting T cells. By Day 57, a mean of 24.1, 16.0 and 31.3 IL-4 secreting T cells were observed per million cells in participants vaccinated with $5 \mu \mathrm{g}$, $15 \mu \mathrm{g}$, and $25 \mu \mathrm{g}$, respectively. The results of cellular immune response are summarized in Fig. 4.

\section{Discussion}

This is the first and currently the only clinical trial report to address the protein-based vaccine using the S-2P protein developed by NIAID, U.S.A. as the antigen, and adjuvanted with CpG 1018 and aluminium hydroxide.

This interim analysis demonstrated that the MVC-COV1901 vaccine was well tolerated and immunogenic in healthy adults aged 20 to 49 years. Across the three dose groups, local injection-site reactions were all mild. This safety profile is similar to that described in the previous report for subunit protein vaccines adjuvanted with CpG 1018 or aluminium hydroxide [9],[10] The rate and severity of solicited AEs (Fig. 2) were similar among the $5 \mu \mathrm{g}, 15 \mu \mathrm{g}$, and $25 \mu \mathrm{g}$ dose groups and between the first and second dose.

There were no neutralizing responses at baseline, aligning with the fact that there was no circulating SARS-CoV-2 in Taiwan. The neutralizing antibody titers were measurable at Day 43 and Day 57 for all dose levels. All participants in the $15 \mu \mathrm{g}$ and $25 \mu \mathrm{g}$ dose groups developed wild-type SARS-CoV-2 neutralizing antibodies and the wild type neutralizing antibody responses correlated well with IgG and pseudo-virus neutralizing antibody titers (Figure S1). Serum neutralizing activity has been shown to be a correlate of protection for other viral vaccines, such as yellow fever vaccine, polio vaccine, and Japanese encephalitis vaccine[11], and is generally accepted as a useful biomarker of the in vivo humoral response. Although the correlate of protection has not yet been demonstrated, neutralizing antibody responses appear to be predictive of immune protection by a COVID19 vaccine [12]. For example, mRNA-1273, with GMT levels 2 3 times[8] the GMT of convalescent sera, demonstrated an efficacy of 94.0\% [6]; BNT162b2, with GMT levels 1.7 4.6 times[13] the value of convalescent sera, demonstrated an efficacy of 95.0\% [14]; and Ad26. COV2.3S, with GMT levels 0.53 0.67 times[15] the value of convalescent sera, demonstrated an efficacy of 70.4\% [16]. The geometric mean titers in the $15 \mu \mathrm{g}$ and $25 \mu \mathrm{g}$ dose groups were generally comparable with those of a panel of control convalescent serum specimens with all participants in both groups seroconverting after two doses. However, the rather low level of GMT for the convalescent samples in this study of 42.7 compared to NIBSC 20/130 at 281.8 suggests these control convalescent samples may not be truly representative. While there are limitations to the use of human convalescent serum because they are likely not representative and there is range of antibody responses based on the severity of illness, the highest titer of GMT 176.5 was still considered comparable with NIBSC 20/ 130 (281.5) for the 2-fold dilution factor in this neutralizing assay. In addition, the preclinical study of MCV-COV1901[17] showed that hamsters were protected from SARS-CoV-2 virus challenge after two doses of $1 \mu \mathrm{g}$ or $5 \mu \mathrm{g}$ S-2P protein adjuvanted with $150 \mu \mathrm{g} \mathrm{CpG}$ and $75 \mu \mathrm{g}$ aluminum hydroxide. Therefore, $15 \mu \mathrm{g}$ S-2P combined with CpG 1018 and aluminum hydroxide was deemed adequate to elicit a profound humoral immune response.

We had also investigated the neutralizing titers of antibodies from sera of 28 days after second dose (Day 57) in this study against pseudoviruses coated with the wildtype, D614G, B.1.1.7, or B.1.351 spike 


\section{A. SARS-CoV-2 spike-specific IgG}

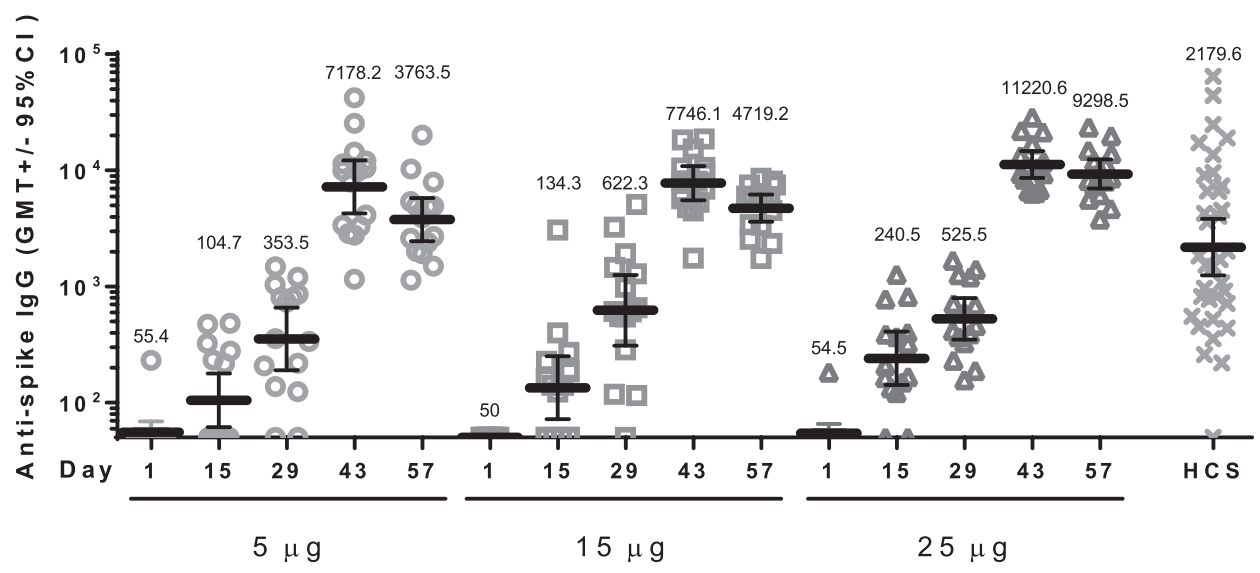

\section{B. SARS-CoV-2 pseudovirus neutralization}

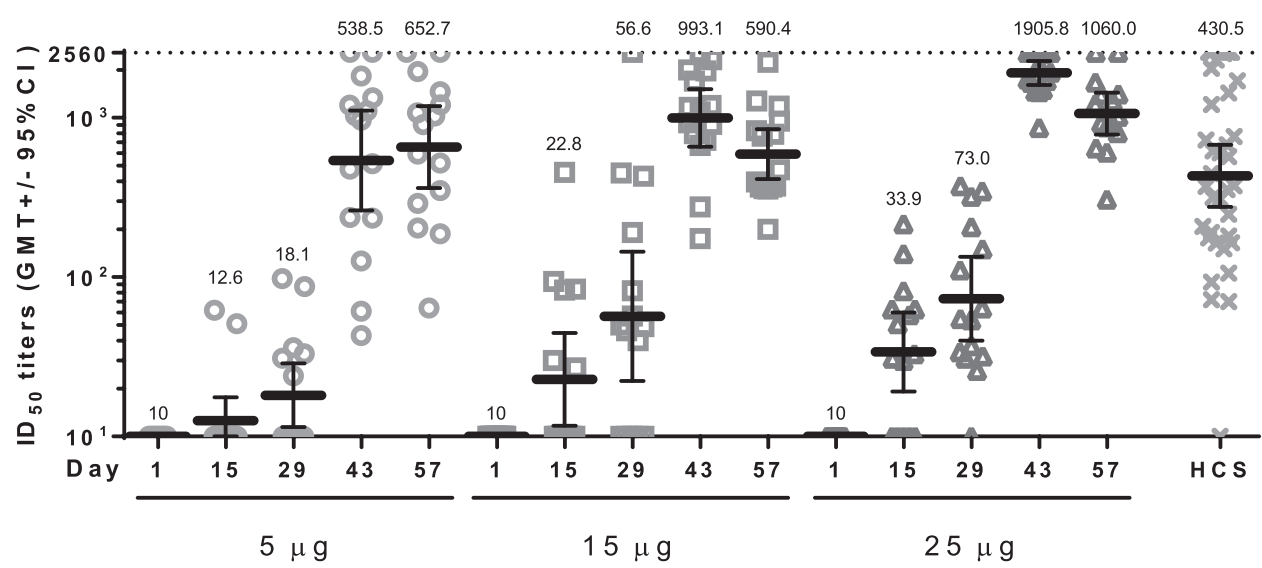

C. Wild-type SARS-CoV-2 neutralization

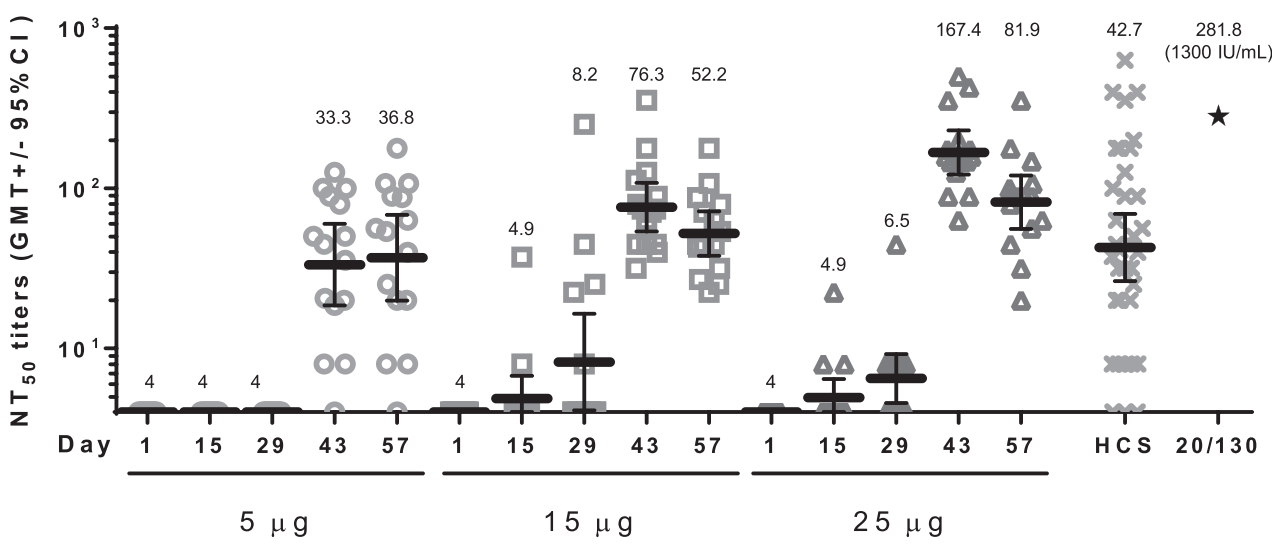

Fig. 3. Summary of Humoral Immune Response

Sera of participants vaccinated with 5, 15, or $25 \mu \mathrm{g}$ of MVC-COV1901 were measured for anti-spike IgG by (A) ELISA, and neutralization titers were measured by (B) pseudovi-

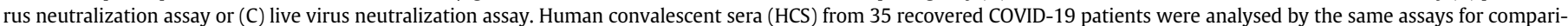

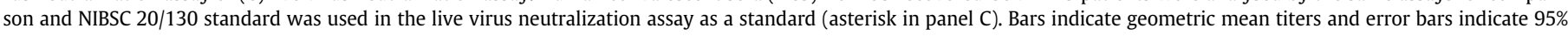

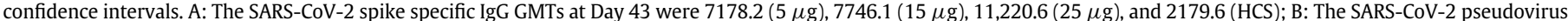

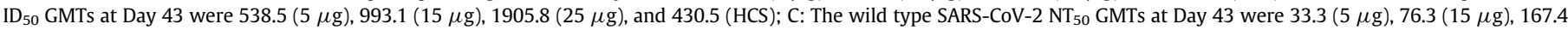
$(25 \mu \mathrm{g})$, and 42.7 (HCS) as detailed in Table S4. The value of NIBSC 20/130 standard was 281.8. (asterisk). 


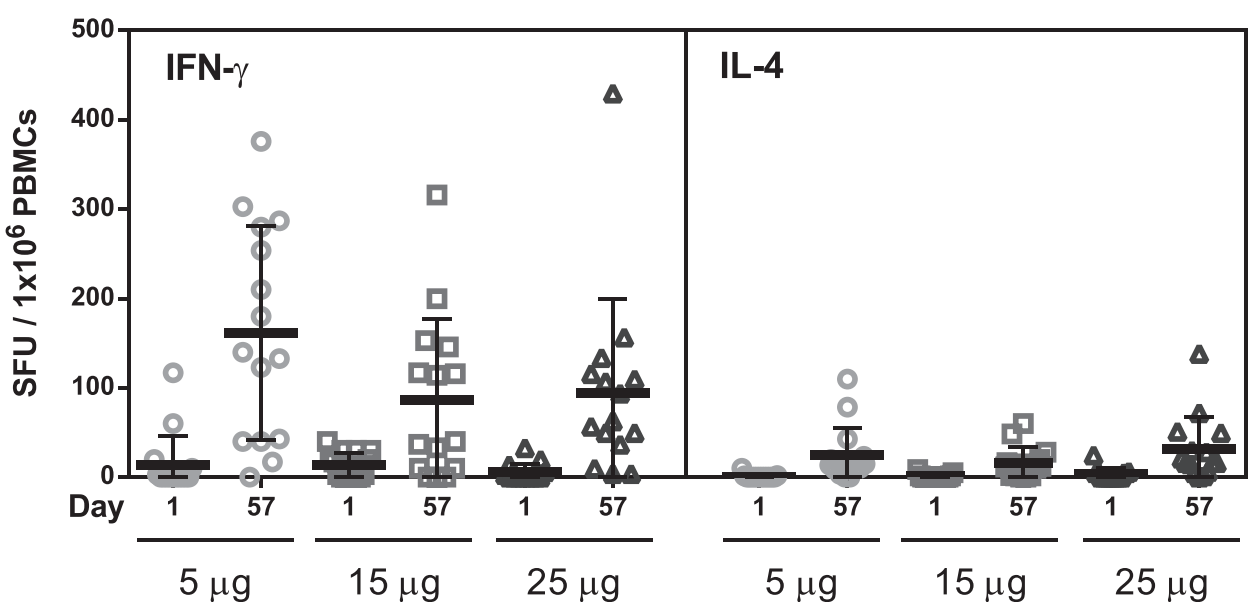

Fig. 4. Summary of Cellular Immune Response

Cells were stimulated with a S1 peptides pool of peptides and incubated at $37^{\circ} \mathrm{C}$ for $24-48 \mathrm{~h}$. Cells stimulated with CD3-2 mAb served as a positive control. IFN- $\gamma$ (left) or

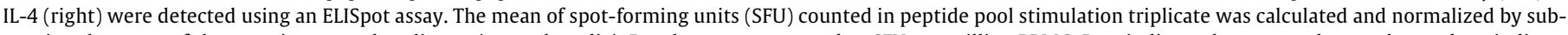

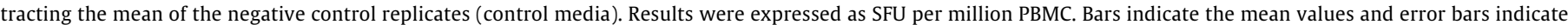
standard deviations.

proteins. The result showed reduced neutralization against the B.1.351 variant and was discussed elsewhere[18].

Previous experience with vaccines and animal models of SARSCoV-1[19] and MERS-CoV[20] infection have raised safety concerns about the potential for Vaccine-Associated Enhanced Disease (VAED). Reducing the risk of VAED by induction of high neutralizing antibody responses and Th1-skewed T-cell responses has become the consensus of COVID-19 vaccine development [21]. Although, it is possible that the observed IFN- $\gamma$ responses were mediated in part by CD8 T cells since the ELISpot assay captures cell-mediated responses indiscriminately. It is worth noting that MVC-COV1901 induced substantially higher numbers of IFN- $\gamma$ - than IL-4- producing cells in human PBMC, suggesting a Th1-skewed immune response.

This interim report has some limitations: the small size of the trial, the short period of follow-up at this time point, and the participants' young age and good health status. We were not able to assess the durability of the immune responses after Day 57 in this interim report. However, participants will be followed up for 6 months after the second dose with scheduled blood collections throughout that period to evaluate humoral immunologic responses.

These safety and immunogenicity findings support further advancement of the MVC-COV1901 vaccine in subsequent clinical trials. Of the three doses evaluated, both the $15 \mu \mathrm{g}$ and $25 \mu \mathrm{g}$ dose elicited high neutralising antibody responses with all participants seroconverting after the second dose and a Th1-skewed $\mathrm{T}$ cell immune response. A phase 2 trial with 3700 participants (including the populations at greatest risk for serious COVID-19 such as those with chronic medical diseases and older adults) is on-going (ClinicalTrials.gov number, NCT04695652).

\section{Author contributions}

SM Hsieh, SC Chang and IC Tai had full access to all of the data in the study and take responsibility for the integrity of the data and the accuracy of the data analysis. SC Chang and IC Tai contributed equally and are joint corresponding authors.

Concept and design: SM Hsieh and IC Tai.

Acquisition, or interpretation of data: SM Hsieh, SC Chang, WD Liu, and YS Huang.

Drafting and preparing the manuscript: SM Hsieh, SC Chang, WD Liu, YS Huang, YJ Lin, EF Hsieh, and IC Tai.

Critical revision of the manuscript for important intellectual content: SM Hsieh, SC Chang, and R Janssen.
Laboratory assays set up and analysis of data: YJ Lin, EF Hsieh, Chung-Guei Hung and Shin-Ru Shih.

Administrative, technical, or material support: Charles Chen and WC Lian.

All authors reviewed and approved of the final version of the manuscript.

\section{Data sharing statement}

Data sharing is not applicable to this article as this is an interim analysis of the study, the trial is still ongoing.

\section{Declaration of Competing Interest}

Szu-Min Hsieh, Shan-Chwen Chang, Wang-Da Liu, Yu-Shan Huang, Chung-Guei Hung and Shin-Ru Shih declared that they have no known competing financial interests or personal relationships that could have appeared to influence the work reported in this paper. Robert Janssen reports personal fees from Dynavax Technologies Corporation, outside the submitted work. Yi-Jiun Lin reports grants from Taiwan Centers for Disease Control, Ministry of Health and Welfare, personal fees from Medigen Vaccine Biologics Corporation, during the conduct of the study. In addition, Yi-Jiun Lin has patent US63/040,696 pending and is an employee of Medigen Vaccine Biologics Corporation. Erh-Fang Hsieh reports grants from Taiwan Centers for Disease Control, Ministry of Health and Welfare, and personal fees from Medigen Vaccine Biologics Corporation, during the conduct of the study. In addition, Erh-Fang Hsieh is an employee of Medigen Vaccine Biologics Corporation. Wei-Cheng Lian reports grants from Taiwan Centers for Disease Control, Ministry of Health and Welfare, and personal fees from Medigen Vaccine Biologics Corporation, during the conduct of the study. In addition, Wei-Cheng Lian is an employee of Medigen Vaccine Biologics Corporation. Charles Chen reports grants from Taiwan Centers for Disease Control, Ministry of Health and Welfare, during the conduct of the study. In addition, Charles Chen has patent US63/040,696 pending. I-Chen Tai reports grants from Taiwan Centers for Disease Control, Ministry of Health and Welfare, and personal fees from Medigen Vaccine Biologics Corporation, during the conduct of the study. In addition, I-Chen Tai is an employee of Medigen Vaccine Biologics Corporation. 


\section{Acknowledgements}

All the participants for their dedication to this trial. Dr. Barney S. Graham, Yaroslav Tsybovsky, Olubukola Abiona, and Kizzmekia S. Corbett at Vaccine Research Center, National Institute of Allergy and Infectious Diseases, U.S.A., team members at Dynavax Technologies, Emeryville, CA 94608, USA. and Tsun-Yung Kuo at Department of Biotechnology and Animal Science, National Ilan University, Ilan, Taiwan for providing technical guidance and helpful advice; The investigational staff at National Taiwan University Hospital, Taiwan and A2 Healthcare Taiwan Corp. for the conduction of the trial; Leo Lee, Tzay Huar Hong, Hui-Yi Wang, Chia En Lien, and Hao-Yuan Cheng at Medigen Vaccine Biologics Corp. for collaboration on protocol development, significant contribution to the Investigational New Drug (IND) application, and participation in weekly meeting with the regulatory authority; The members of the safety monitoring committee; Dr. YuChi Chou and his team members at the RNAi Core Facility, Academia Sinica for the pseudovirus neutralization assay; Team members at TFBS Bioscience Incorporation, Taiwan for cellular immune response assay; Team members at Protech Pharmaservices Corporation for spike specific IgG ELISA assay. Dr. Chia En Lien, Dr. Meei-Yun Lin, Dr. Luke Tzu-Chi Liu, Meng-Ju Tsai at Medigen Vaccine Biologics Corp., Taiwan for manuscript editing and revision.

\section{Funding}

Medigen Vaccine Biologics Corporation.

\section{Supplementary materials}

Supplementary material associated with this article can be found, in the online version, at doi:10.1016/j.eclinm.2021.100989.

\section{References}

[1] World Health Organization. WHO Director-General's Opening Remarks at the Mission Briefing on COVID-19-11 March 2020. World Health Organization; March 11, 2020 https://www.who.int/director-general/speeches/detail/whodirector-general-s-opening-remarks-at-the-media-briefing-on-covid- 19-11march-2020 (accessed March 3, 2021).

[2] World Health Organization. COVID-19 Clinical Management: Living Guidance, 25 January 2021. World Health Organization. January 25, 2021. https://www.who. int/publications/i/item/WHO-2019-nCoV-clinical-2021-1 (accessed March 3, 2021).
[3] Graham BS. Rapid COVID-19 vaccine development. Science 2020;368:945-6.

[4] Wrapp D, Wang N, Corbett KS, et al. Cryo-EM structure of the 2019-nCoV spike in the prefusion conformation. Science 2020;367:1260-3.

[5] Pallesen J, Wang N, Corbett KS, et al. Immunogenicity and structures of a rationally designed prefusion MERS-CoV spike antigen. Proc Natl Acad Sci USA 2017:114:E7348-57.

[6] Baden LR, El Sahly HM, Essink B, et al. Efficacy and safety of the mRNA-1273 SARS-CoV-2 Vaccine. N Engl J Med 2021;384:403-16.

[7] Kuo T-Y, Lin M-Y, Coffman RL, et al. Development of CpG-adjuvanted stable prefusion SARS-CoV-2 spike antigen as a subunit vaccine against COVID-19. Sci Rep 2020;10. doi: 10.1038/s41598-020-77077-z.

[8] Jackson LA, Anderson EJ, Rouphael NG, et al. An mRNA vaccine against SARS-CoV2 - preliminary report. N Engl J Med 2020;383:1920-31.

[9] HEPLISAV-B. [Hepatitis b Vaccine (Recombinant), Adjuvanted]. Package Insert. Dynavax Technologies Corporation; 2020.

[10] Mullen GED, Ellis RD, Miura K, et al. Phase 1 Trial of AMA1-C1/Alhydrogel plus CPG 7909: an Asexual Blood-Stage Vaccine for Plasmodium falciparum Malaria. PLoS ONE 2008;3:e2940.

[11] U.S. Food and Drug Administration. Table of Surrogate Endpoints That Were the Basis of Drug Approval or Licensure. U.S. Food and Drug Administration; August 20, 2020 https://www.fda.gov/drugs/development-resources/table-surrogateendpoints-were-basis-drug-approval-or-licensure (accessed March 3, 2021).

[12] Khoury DS, Cromer D, Reynaldi A, et al. Neutralizing antibody levels are highly predictive of immune protection from symptomatic SARS-CoV-2 infection. Nat Med 2021. doi: 10.1038/s41591-021-01377-8.

[13] Walsh EE, Frenck Jr RW, Falsey AR, et al. Safety and immunogenicity of two RNAbased Covid-19 vaccine candidates. N Engl J Med 2020;383:2439-50.

[14] Polack FP, Thomas SJ, Kitchin N, et al. Safety and efficacy of the BNT162b2 mRNA Covid-19 Vaccine. N Engl J Med 2020;383:2603-15.

[15] Folegatti PM, Ewer KJ, Aley PK, et al. Safety and immunogenicity of the ChAdOx1 nCoV-19 vaccine against SARS-CoV-2: a preliminary report of a phase $1 / 2$, singleblind, randomised controlled trial. The Lancet 2020;396:467-78.

[16] Voysey M, Clemens SAC, Madhi SA, et al. Safety and efficacy of the ChAdOx1 nCoV-19 vaccine (AZD1222) against SARS-CoV-2: an interim analysis of four randomised controlled trials in Brazil, South Africa, and the UK. The Lancet 2021;397:99-111.

[17] Lien CE, Lin YJ, Kuo TY, et al. CpG-adjuvanted stable prefusion SARS-CoV-2 spike protein protected hamsters from SARS-CoV-2 challenge. Sci Rep 2021;11:8761. doi: 10.1038/s41598-021-88283-8.

[18] Lien C.E., Kuo T.Y., Lin Y.J., et al. Evaluating the neutralizing ability of a CpG-adjuvanted S-2P subunit vaccine against SARS-CoV-2 Variants of Concern. MedRixv. Published online Mar 22, 2021. https://doi.org/10.1101/2021.03.19.21254000

[19] Tseng C-T, Sbrana E, Iwata-Yoshikawa N, et al. Immunization with SARS Coronavirus vaccines leads to pulmonary immunopathology on challenge with the SARS Virus. PLoS ONE 2012;7:e35421.

[20] Agrawal AS, Tao X, Algaissi A, et al. Immunization with inactivated Middle East Respiratory Syndrome coronavirus vaccine leads to lung immunopathology on challenge with live virus. Hum Vaccin Immunother 2016;12:2351-6.

[21] Lambert P-H, Ambrosino DM, Andersen SR, et al. Consensus summary report for CEPI/BC March 12-13, 2020 meeting: assessment of risk of disease enhancement with COVID-19 vaccines. Vaccine 2020;38:4783-91. 